\title{
Systems of semilinear evolution inequalities with temporal fractional derivative on the Heisenberg group
}

Bekkar Meneceur ${ }^{1,2}$, Kamel Haouam $^{3}$ and Amar Debbouche ${ }^{4^{*}}$

\author{
"Correspondence: \\ amar_debbouche@yahoo.fr \\ ${ }^{4}$ Department of Mathematics, \\ Guelma University, Guelma, 24000, \\ Algeria \\ Full list of author information is \\ available at the end of the article
}

\section{Abstract \\ We investigate nonexistence results of nontrivial solutions of fractional differential inequalities of the form

$$
\left(\mathrm{FS}_{q}^{m}\right):\left\{\begin{array}{l}
\left.\mathbf{D}_{0 / t}^{q} x_{i}-\Delta_{\mathbb{H}}\left(\lambda_{i} x_{i}\right) \geq|\eta|^{\alpha_{i+1}}\left|x_{i+1}\right|^{\beta_{i+1}}, \quad(\eta, t) \in \mathbb{H}^{N} \times\right] 0,+\infty[, 1 \leq i \leq m, \\
x_{m+1}=x_{1}
\end{array}\right.
$$

where $\mathbf{D}_{0 / t}^{q}$ is the time-fractional derivative of order $q \in(1,2)$ in the sense of Caputo, $\Delta_{\mathbb{H}}$ is the Laplacian in the $(2 N+1)$-dimensional Heisenberg group $\mathbb{H}^{N},|\eta|$ is the distance from $\eta$ in $\mathbb{H}^{N}$ to the origin, $m \geq 2, \alpha_{m+1}=\alpha_{1}, \beta_{m+1}=\beta_{1}$, and $\lambda_{i} \in L^{\infty}\left(\mathbb{H}^{N} \times\right] 0,+\infty[), 1 \leq i \leq m$. The main results are concerned with $Q \equiv 2 N+2$, less than the critical exponents that depend on $q, \alpha_{i}$, and $\beta_{i}, 1 \leq i \leq m$. For $q=2$, we deduce the results given by El Hamidi and Kirane (Abstr. Appl. Anal. 2004(2):155-164, 2004) and El Hamidi and Obeid (J. Math. Anal. Appl. 208(1):77-90, 2003) from the hyperbolic systems. For $m=1$, we study the scalar case

$$
\left(\mathrm{FI}_{q}\right): \quad \mathbf{D}_{0 / t}^{q} x-\Delta_{\mathbb{H}}(\lambda x) \geq|\eta|^{\alpha}|x|^{\beta},
$$

where $\beta>1, \alpha$ are real parameters. In the last case, for $q=2$, we return to the approach of Pohozaev and Véron (Manuscr. Math. 102:85-99, 2000) from the hyperbolic inequalities.

MSC: 35A01; 35B33; 35R03; 35R11; 35R45

Keywords: critical exponent; fractional derivative; Heisenberg group; evolution inequalities; test function method

\section{Introduction}

Pohozaev and Véron [3] have established the question of nonexistence results for solutions of semilinear hyperbolic inequalities of the type

$$
\frac{\partial^{2} x}{\partial t^{2}}-\Delta_{\mathbb{H}}(\lambda x) \geq|\eta|_{\mathbb{H}}^{\alpha}|x|^{\beta}
$$

it is shown that no weak solution $x$ exists provided that

$$
\int_{\mathbb{R}^{2 N+1}} x_{1}(\eta) d \eta \geq 0, \quad \alpha>-2 \quad \text { and } \quad 1<\beta \leq \frac{Q+1+\alpha}{Q-1}
$$

(c) The Author(s) 2017. This article is distributed under the terms of the Creative Commons Attribution 4.0 International License (http://creativecommons.org/licenses/by/4.0/), which permits unrestricted use, distribution, and reproduction in any medium, provided you give appropriate credit to the original author(s) and the source, provide a link to the Creative Commons license, and indicate if changes were made. 
In [1], El Hamidi and Kirane presented analogous results for a system of $m$ hyperbolic semilinear inequalities of the form

$$
\left(\mathrm{HS}^{m}\right):\left\{\begin{array}{l}
\frac{\partial^{2} x_{i}}{\partial t^{2}}-\Delta_{\mathbb{H}}\left(\lambda_{i} x_{i}\right) \geq|\eta|^{\alpha_{i+1}}\left|x_{i+1}\right|^{\beta_{i+1}}, \\
\left.(\eta, t) \in \mathbb{H}^{N} \times\right] 0,+\infty[, \quad 1 \leq i \leq m, \\
x_{m+1}=x_{1}
\end{array}\right.
$$

and expressed the Fujita exponent (see [4-6]), which ensures the system $\left(\mathrm{HS}^{m}\right)$ admits no solution defined in $\mathbb{H}^{N}$ whenever $Q \leq 1+\max \left(X_{1}, X_{2}, \ldots, X_{m}\right)$, where $\left(X_{1}, X_{2}, \ldots, X_{m}\right)^{T}$ for the solution of the linear system (27).

Their results have been generalized by El Hamidi and Obeid [2] to a system of $m$ semilinear inequalities with higher-order time derivative of the type

$$
\left(S_{k}^{m}\right):\left\{\begin{array}{l}
\frac{\partial^{k} x_{i}}{\partial t^{k}}-\Delta_{\mathbb{H}}\left(\lambda_{i} x_{i}\right) \geq|\eta|^{\alpha_{i+1}}\left|x_{i+1}\right|^{\beta_{i+1}}, \\
\left.(\eta, t) \in \mathbb{H}^{N} \times\right] 0,+\infty[, \quad 1 \leq i \leq m, \\
x_{m+1}=x_{1}, \quad k=1,2, \ldots
\end{array}\right.
$$

where they proved that the system $\left(S_{k}^{m}\right)$ admits no solution defined in $\mathbb{H}^{N}$ whenever $Q \leq$ $2\left(1-\frac{1}{k}\right)+\max \left(X_{1}, X_{2}, \ldots, X_{m}\right)$. Different works on the importance of inequalities can be found in $[7,8]$.

In this paper, we generalize these results $\left(\right.$ for $\left.\left(\mathrm{HS}^{m}\right)\right)$ to an evolution system with temporal fractional derivative of the form

$$
\left(\mathrm{FS}_{q}^{m}\right):\left\{\begin{array}{l}
\mathbf{D}_{0 / t}^{q} x_{i}-\Delta_{\mathbb{H}}\left(\lambda_{i} x_{i}\right) \geq|\eta|^{\alpha_{i+1}\left|x_{i+1}\right|^{\beta_{i+1}}} \\
\left.(\eta, t) \in \mathbb{H}^{N} \times\right] 0,+\infty[, \quad 1 \leq i \leq m, \\
x_{m+1}=x_{1} q \in(1,2)
\end{array}\right.
$$

and we show under certain initial conditions that the system $\left(\mathrm{FS}_{q}^{m}\right)$ admits no solution defined in $\mathbb{H}^{N}$ whenever $Q<Q_{q}^{\bullet}=2\left(1-\frac{1}{q}\right)+\max \left(X_{1}, X_{2}, \ldots, X_{m}\right)$.

This paper is organized as follows. In Section 2, we present some essential facts from fractional calculus, more precisely, the definitions of the fractional derivative in the sense of Riemann-Liouville and in sense of Caputo and their relationship between them, for some new senses: the reader may refer to [9-11]. We also give some preliminaries as regards the Heisenberg group $\mathbb{H}^{N}$ and the operator $\Delta_{\mathbb{H}}$. In Section 3, we study the case of two inequalities. In Section 4, we study the general case of $m>2$, and in the last Section 5, we study the scalar case.

\section{Notation and preliminaries}

In this section, we present some known facts about the time-fractional derivative $\mathbf{D}_{0 / t}^{q}$, the Heisenberg group $\mathbb{H}^{N}$ and the operator $\Delta_{\mathbb{H}}$.

The left-sided derivative and the right-sided derivative in the sense of Riemann-Liouville for $\psi \in L^{1}(0, T)$, of order $q \in(1,2)$ are defined, respectively, as follows:

$$
\begin{aligned}
& \left(D_{0 / t}^{q} \psi\right)(t)=\frac{1}{\Gamma(2-q)}\left(\frac{d}{d t}\right)^{2} \int_{0}^{t} \frac{\psi(\sigma)}{(t-\sigma)^{q-1}} d \sigma, \\
& \left(D_{t / T}^{q} \psi\right)(t)=\frac{1}{\Gamma(2-q)}\left(\frac{d}{d t}\right)^{2} \int_{t}^{T} \frac{\psi(\sigma)}{(\sigma-t)^{q-1}} d \sigma,
\end{aligned}
$$

where $\Gamma$ is the Euler gamma function. 
If $\psi^{\prime \prime} \in L^{1}(0, T)$, the derivative in the sense of Caputo of order $q \in(1,2)$ is defined by

$$
\left(\mathbf{D}_{0 / t}^{q} \psi\right)(t)=\frac{1}{\Gamma(2-q)} \int_{0}^{t} \frac{\psi^{\prime \prime}(\sigma)}{(t-\sigma)^{q-1}} d \sigma
$$

which is related to the Riemann-Liouville derivative by

$$
\mathbf{D}_{0 / t}^{q} \psi(t)=D_{0 / t}^{q}\left(\psi(t)-\psi(0)-t \psi^{\prime}(0)\right)
$$

We also recall the formula of integration by parts if $0<\delta<1$ :

$$
\int_{0}^{T} \varphi(t)\left(D_{0 / t}^{\delta} \psi\right)(t) d t=\int_{0}^{T}\left(D_{t / T}^{\delta} \varphi\right)(t) \psi(t) d t
$$

To derive the weak formulations, we have made use of the relations (see (2.30) and (2.31), p.37 in[12]):

$$
\begin{aligned}
& D_{o / t}^{1+q} \psi=D D_{o / t}^{q} \psi, \quad q \in(0,1), \\
& D_{t / T}^{1+q} \psi=-D D_{t / T}^{q} \psi, \quad q \in(0,1),
\end{aligned}
$$

we also have the following formula (see Lemma 2.2, p.35 in [12]), for any $\delta \in(0,1)$ :

$$
D_{t / T}^{\delta} \psi(t)=\frac{1}{\Gamma(1-\delta)}\left(\frac{\psi(T)}{(T-t)^{\delta}}-\int_{t}^{T} \frac{\psi^{\prime}(\sigma)}{(\sigma-t)^{\delta}} d \sigma\right)
$$

More details of fractional derivatives can be found in $[5,12,13]$; see also [14-16].

The Heisenberg group $\mathbb{H}^{n}$ of the dimension $(2 N+1)$ is the space

$$
\mathbb{R}^{2 N+1}=\left\{\eta=(x, y, \tau) \in \mathbb{R}^{N} \times \mathbb{R}^{N} \times \mathbb{R}\right\}
$$

equipped with the group operation 'o' defined by

$$
\eta \circ \tilde{\eta}=\left(x+\tilde{x}, y+\tilde{y}, \tau+\tilde{\tau}+2 \sum_{i=1}^{N}\left(x_{i} \tilde{y}_{i}-\tilde{x}_{i} y_{i}\right)\right),
$$

where

$$
\begin{aligned}
& \eta=(x, y, \tau)=\left(x_{1}, x_{2}, \ldots, x_{N}, y_{1}, y_{2}, \ldots, y_{N}, \tau\right), \\
& \tilde{\eta}=(\tilde{x}, \tilde{y}, \tilde{\tau})=\left(\tilde{x}_{1}, \tilde{x}_{2}, \ldots, \tilde{x}_{N}, \tilde{y}_{1}, \tilde{y}_{2}, \ldots, \tilde{y}_{N}, \tilde{\tau}\right),
\end{aligned}
$$

this group operation makes $\mathbb{H}^{n}$ have the structure of a Lie group.

The subelliptic Laplacian $\Delta_{\mathbb{H}}$ over $\mathbb{H}^{n}$ is defined by

$$
\Delta_{\mathbb{H}}=\sum_{i=1}^{N}\left(X_{i}^{2}+Y_{i}^{2}\right)
$$


where

$$
X_{i}=\frac{\partial}{\partial x_{i}}+2 y_{i} \frac{\partial}{\partial \tau} \quad \text { and } \quad Y_{i}=\frac{\partial}{\partial y_{i}}-2 x_{i} \frac{\partial}{\partial \tau}
$$

with a simple calculation, we can write

$$
\Delta_{\mathbb{H}}=\sum_{i=1}^{N}\left(\frac{\partial^{2}}{\partial x_{i}^{2}}+\frac{\partial^{2}}{\partial y_{i}^{2}}+4 y_{i} \frac{\partial^{2}}{\partial x_{i} \partial \tau}-4 x_{i} \frac{\partial^{2}}{\partial y_{i} \partial \tau}+4\left(x_{i}^{2}+y_{i}^{2}\right) \frac{\partial^{2}}{\partial \tau^{2}}\right) .
$$

The operator $\Delta_{\mathbb{H}}$ is a degenerate elliptic operator satisfying the Hörmander condition of order 1 (see [17]). It is invariant with respect to the left multiplication in the group since

$$
\Delta_{\mathbb{H}}(x(\eta \circ \tilde{\eta}))=\left(\Delta_{\mathbb{H}} x\right)(\eta \circ \tilde{\eta}) \quad \forall(\eta, \tilde{\eta}) \in \mathbb{H}^{N} \times \mathbb{H}^{N} .
$$

The distance between a point and the origin in $\mathbb{H}^{N}$ is defined by

$$
|\eta|_{\mathbb{H}}=\left(\tau^{2}+\sum_{i=1}^{N}\left(x_{i}^{2}+y_{i}^{2}\right)^{2}\right)^{1 / 4}
$$

The application $\eta \rightarrow|\eta|_{\mathbb{H}}$ is homogeneous of degree one with respect to the natural group of dilatations

$$
\delta_{\lambda}(\eta)=\left(\lambda x, \lambda y, \lambda^{2} t\right)
$$

We also know that the operator $\Delta_{\mathbb{H}}$ is homogeneous of degree 2 relative to the distance $\delta_{\lambda}$ given in (11), that is,

$$
\Delta_{\mathbb{H}}=\lambda^{2} \delta_{\lambda}\left(\Delta_{\mathbb{H}}\right)
$$

Obviously, the action of $\Delta_{\mathbb{H}}$ where the functions only depend on $\rho=|\eta|_{\mathbb{H}}$ is

$$
\Delta_{\mathbb{H}} x(\rho)=a(\eta)\left(\frac{d^{2} x}{d \rho^{2}}+\frac{(Q-1)}{\rho} \frac{d x}{d \rho}\right)
$$

where

$$
a(\eta)=\sum_{i=1}^{N} \frac{\left(x_{i}^{2}+y_{i}^{2}\right)}{\rho^{2}} \quad \text { and } \quad Q=2 N+2 .
$$

The number $Q$ defined above is called the homogeneous dimension $\mathbb{H}^{N}$.

We also identify the points $\mathbb{H}^{N}$ with those of $\mathbb{R}^{2 N+1}$, and we refer to the natural measurement of Hâar in $\mathbb{H}^{N}$ similar to that of Lebesgue $d \eta=d x d y d \tau$ in $\mathbb{R}^{2 N+1}$. Readers can refer to [17-22] for more details of the analysis of the Heisenberg group. 


\section{Systems of two inequalities}

In this section, we are interested with systems of type

$$
\left(\mathrm{FS}_{q}^{2}\right): \begin{cases}\mathbf{D}_{0 / t}^{q} x-\Delta_{\mathbb{H}}\left(\lambda_{1} x\right) \geq|\eta|_{\mathbb{H}}^{\alpha_{1}}|y|^{\beta_{1}} & \text { in } \mathbb{H}^{n} \times \mathbb{R}^{+} \\ \mathbf{D}_{0 / t}^{q} y-\Delta_{\mathbb{H}}\left(\lambda_{2} y\right) \geq|\eta|_{\mathbb{H}}^{\alpha_{2}}|x|^{\beta_{2}} & \text { in } \mathbb{H}^{n} \times \mathbb{R}^{+}\end{cases}
$$

where $\mathbf{D}_{0 / t}^{q}$ denotes the time-fractional derivative of order $q \in(1,2)$, in the sense of Caputo. The functions $\lambda_{1}$ and $\lambda_{2}$ introduced in (12) are assumed to be measurable and bounded functions on $\mathbb{H}^{n} \times \mathbb{R}^{+}$, where the exponents $\alpha_{1}, \alpha_{2}$ and $\beta_{1}, \beta_{2}>1$ are real numbers. We denote by $D_{0 / t}^{q}$, the time-fractional derivative of order $q \in(1,2)$ in the sense of RiemannLiouville. The following holds.

Definition 3.1 Let $\lambda_{1}$ and $\lambda_{2}$ be two bounded measurable functions in $Q_{T}=\mathbb{R}^{2 N+1} \times$ $(0, T)$. A weak solution $(x, y)$ of the system $\left(\mathrm{FS}_{q}^{2}\right)$ with positive initial data $x_{0}, x_{1}, y_{0}, y_{1} \in$ $L_{\text {loc }}^{1}\left(\mathbb{R}^{2 N+1}\right)$ is a pair of locally integrable functions $(x, y)$ such that $(x, y) \in L^{\beta_{2}}\left(Q_{T}\right.$, $\left.|\eta|_{\mathbb{H}}^{\alpha_{2}} d \eta d t\right) \times L^{\beta_{1}}\left(Q_{T},|\eta|_{\mathbb{H}}^{\alpha_{1}} d \eta d t\right)$ satisfying

$$
\left\{\begin{array}{l}
\int_{Q_{T}}\left(-x D_{t / T}^{q} \varphi+\lambda_{1} x \Delta_{\mathbb{H}} \varphi+|\eta|_{\mathbb{H}}^{\alpha_{1}}|y|^{\beta_{1}} \varphi+x_{1}(\eta) D_{t / T}^{q-1} \varphi\right) d \eta d t \\
\quad+\int_{\mathbb{R}^{2 N+1}} x_{0}(\eta) D_{t / T}^{q-1} \varphi(0) d \eta \leq 0, \\
\int_{Q_{T}}\left(-y D_{t / T}^{q} \varphi+\lambda_{2} y \Delta_{\mathbb{H}} \varphi+|\eta|_{\mathbb{H}}^{\alpha_{2}}|x|^{\beta_{2}} \varphi+y_{1}(\eta) D_{t / T}^{q-1} \varphi\right) d \eta d t \\
\quad+\int_{\mathbb{R}^{2 N+1}} y_{0}(\eta) D_{t / T}^{q-1} \varphi(0) d \eta \leq 0
\end{array}\right.
$$

for any nonnegative test function $\varphi \in C_{c}^{2}\left(Q_{T}\right)$, such that $\varphi(\cdot, T)=D_{t / T}^{q-1} \varphi(\cdot, T)=0$.

Remark 3.2 We assume that the integrals in (13) are convergent. In Definition 3.1, if $T=$ $+\infty$, then the solution is called global.

Theorem 3.3 Assume that

$$
Q<Q_{q}^{\bullet}=2\left(1-\frac{1}{q}\right)+\frac{1}{\beta_{1} \beta_{2}-1} \max \left(\left(\alpha_{1}+2\right)+\beta_{1}\left(\alpha_{2}+2\right), \beta_{2}\left(\alpha_{1}+2\right)+\left(\alpha_{2}+2\right)\right) .
$$

Then there is no weak nontrivial solution $(x, y)$ of the system $\left(\mathrm{FS}_{q}^{2}\right)$.

Proof By contradiction, we suppose $(x, y)$ to be a nontrivial weak solution of $\left(\mathrm{FS}_{q}^{2}\right)$, which generally exists in time, that is, $(x, y)$ exists in $\left(0, T^{*}\right)$ for an arbitrary $T^{*}$.

Let $T$ and $R$ be two positive real numbers such that $0<T R<T^{*}$.

Since the initial data $x_{0}, x_{1}, y_{0}, y_{1}$ are nonnegative, and $D_{t / T}^{q-1} \varphi \geq 0$ (from (8)), the variational formulation (13) implies

$$
\left\{\begin{array}{l}
\int_{Q_{T R}}|\eta|_{\mathbb{H}}^{\alpha_{1}}|y|^{\beta_{1}} \varphi d \eta d t \leq \int_{Q_{T R}} x D_{t / T R}^{q} \varphi d \eta d t-\int_{Q_{T R}} \lambda_{1} x \Delta_{\mathbb{H}} \varphi d \eta d t, \\
\int_{Q_{T R}}|\eta|_{\mathbb{H}}^{\alpha_{2}}|x|^{\beta_{2}} \varphi d \eta d t \leq \int_{Q_{T R}} y D_{t / T R}^{q} \varphi d \eta d t-\int_{Q_{T R}} \lambda_{2} y \Delta_{\mathbb{H}} \varphi d \eta d t .
\end{array}\right.
$$


From the Hölder inequality, we get

$$
\left\{\begin{array}{l}
\int_{Q_{T R}}|\eta|_{\mathbb{H}}^{\alpha_{1}}|y|^{\beta_{1}} \varphi d \eta d t \\
\leq\left(\int_{Q_{T R}}|\eta|_{\mathbb{H}}^{\alpha_{2}}|x|^{\beta_{2}} \varphi d \eta d t\right)^{\frac{1}{\beta_{2}}}\left(\int_{Q_{T R}}\left|D_{t / T R}^{q} \varphi\right|^{\beta_{2}^{\prime}}\left(|\eta|_{\mathbb{H}}^{\alpha_{2}} \varphi\right)^{-\frac{\beta_{2}^{\prime}}{\beta_{2}}} d \eta d t\right)^{\frac{1}{\beta_{2}^{\prime}}} \\
\quad+\left\|\lambda_{1}\right\|_{\infty}\left(\int_{Q_{T R}}|\eta|_{\mathbb{H}}^{\alpha_{2}}|x|^{\beta_{2}} \varphi d \eta d t\right)^{\frac{1}{\beta_{2}}}\left(\int_{Q_{T R}}\left|\Delta_{\mathbb{H}} \varphi\right|^{\beta_{2}^{\prime}}\left(|\eta|_{\mathbb{H}}^{\alpha_{2}} \varphi\right)^{-\frac{\beta_{2}^{\prime}}{\beta_{2}}} d \eta d t\right)^{\frac{1}{\beta_{2}^{\prime}}}
\end{array}\right.
$$

and

$$
\left\{\begin{array}{l}
\int_{Q_{T R}}|\eta|_{\mathbb{H}}^{\alpha_{2}}|x|^{\beta_{2}} \varphi d \eta d t \\
\leq\left(\int_{Q_{T R}}|\eta|_{\mathbb{H}}^{\alpha_{1}}|y|^{\beta_{1}} \varphi d \eta d t\right)^{\frac{1}{\beta_{1}}}\left(\int_{Q_{T R}}\left|D_{t / T R}^{q} \varphi\right|^{\beta_{1}^{\prime}}\left(|\eta|_{\mathbb{H}}^{\alpha_{1}} \varphi\right)^{-\frac{\beta_{1}^{\prime}}{\beta_{1}}} d \eta d t\right)^{\frac{1}{\beta_{1}^{\prime}}} \\
\quad+\left\|\lambda_{2}\right\|_{\infty}\left(\int_{Q_{T R}}|\eta|_{\mathbb{H}}^{\alpha_{1}}|y|^{\beta_{1}} \varphi d \eta d t\right)^{\frac{1}{\beta_{1}}}\left(\int_{Q_{T R}}\left|\Delta_{\mathbb{H}} \varphi\right|^{\beta_{1}^{\prime}}\left(|\eta|_{\mathbb{H}}^{\alpha_{1}} \varphi\right)^{-\frac{\beta_{1}^{\prime}}{\beta_{1}}} d \eta d t\right)^{\frac{1}{\beta_{1}^{\prime}}} .
\end{array}\right.
$$

Next, $C$ denotes a constant which may vary from line to line but is independent on the terms which will take part in any limit process. So, we obtain

$$
\int_{Q_{T R}}|\eta|_{\mathbb{H}}^{\alpha_{1}}|y|^{\beta_{1}} \varphi d \eta d t \leq C\left(\int_{Q_{T R}}|\eta|_{\mathbb{H}}^{\alpha_{2}}|x|^{\beta_{2}} \varphi d \eta d t\right)^{\frac{1}{\beta_{2}}} \mathcal{A}
$$

and

$$
\int_{Q_{T R}}|\eta|_{\mathbb{H}}^{\alpha_{2}}|x|^{\beta_{2}} \varphi d \eta d t \leq C\left(\int_{Q_{T R}}|\eta|_{\mathbb{H}}^{\alpha_{1}}|y|^{\beta_{1}} \varphi d \eta d t\right)^{\frac{1}{\beta_{1}}} \mathcal{B}
$$

where

$$
\begin{aligned}
& \mathcal{A}=\left(\int_{Q_{T R}}\left|D_{t / T R}^{q} \varphi\right|^{\beta_{2}^{\prime}}\left(|\eta|_{\mathbb{H}}^{\alpha_{2}} \varphi\right)^{-\frac{\beta_{2}^{\prime}}{\beta_{2}}} d \eta d t\right)^{\frac{1}{\beta_{2}^{\prime}}}+\left(\int_{Q_{T R}}\left|\Delta_{\mathbb{H}} \varphi\right|^{\beta_{2}^{\prime}}\left(|\eta|_{\mathbb{H}}^{\alpha_{2}} \varphi\right)^{-\frac{\beta_{2}^{\prime}}{\beta_{2}}} d \eta d t\right)^{\frac{1}{\beta_{2}^{\prime}}}, \\
& \mathcal{B}=\left(\int_{Q_{T R}}\left|D_{t / T R}^{q} \varphi\right|^{\beta_{1}^{\prime}}\left(|\eta|_{\mathbb{H}}^{\alpha_{1}} \varphi\right)^{-\frac{\beta_{1}^{\prime}}{\beta_{1}}} d \eta d t\right)^{\frac{1}{\beta_{1}^{\prime}}}+\left(\int_{Q_{T R}}\left|\Delta_{\mathbb{H}} \varphi\right|^{\beta_{1}^{\prime}}\left(|\eta|_{\mathbb{H}}^{\alpha_{1}} \varphi\right)^{-\frac{\beta_{1}^{\prime}}{\beta_{1}}} d \eta d t\right)^{\frac{1}{\beta_{1}^{\prime}}}
\end{aligned}
$$

from (14), (15), we have

$$
\begin{aligned}
& \left(\int_{Q_{T R}}|\eta|_{\mathbb{H}}^{\alpha_{1}}|y|^{\beta_{1}} \varphi d \eta d t\right)^{1-\frac{1}{\beta_{1} \beta_{2}}} \leq C \mathcal{B}^{\frac{1}{\beta_{2}}} \mathcal{A}, \\
& \left(\int_{Q_{T R}}|\eta|_{\mathbb{H}}^{\alpha_{2}}|x|^{\beta_{2}} \varphi d \eta d t\right)^{1-\frac{1}{\beta_{1} \beta_{2}}} \leq C \mathcal{A}^{\frac{1}{\beta_{1}}} \mathcal{B} .
\end{aligned}
$$

Now, we take

$$
\varphi(\eta, t)=\varphi(x, y, \tau, t)=\Phi\left(\frac{\tau^{2 \theta}+|x|^{4 \theta}+|y|^{4 \theta}+t^{4}}{R^{4}}\right),
$$


where $\Phi \in \mathcal{D}\left(\mathbb{R}^{+}\right)$is a smooth nonnegative test function which satisfies $0 \leq \Phi \leq 1$ and

$$
\Phi(r)= \begin{cases}0 & \text { if } r \geq 2 \\ 1 & \text { if } 0 \leq r \leq 1\end{cases}
$$

Then $\theta>1$, which will be specified later.

Then

$$
\left\{\begin{aligned}
\Delta_{\mathbb{H}} \varphi(\eta, t)= & \frac{4 \theta \Phi^{\prime}(\rho)}{R^{4}}\left[(N+2(2 \theta-1))\left(|x|^{2(2 \theta-1)}+|y|^{2(2 \theta-1)}\right)\right. \\
& \left.+2(2 \theta-1) \tau^{2(\theta-1)}\left(|x|^{2}+|y|^{2}\right)\right] \\
& +\frac{16 \theta^{2} \Phi^{\prime \prime}(\rho)}{R^{8}}\left[|x|^{2(4 \theta-1)}+|y|^{2(4 \theta-1)}+2 \tau^{2 \theta-1}\langle x, y\rangle\left(|x|^{2(2 \theta-1)}-|y|^{2(2 \theta-1)}\right)\right. \\
& \left.+\tau^{2(2 \theta-1)}\left(|x|^{2}+|y|^{2}\right)\right]
\end{aligned}\right.
$$

where

$$
\rho=\frac{\tau^{2 \theta}+|x|^{4 \theta}+|y|^{4 \theta}+t^{4}}{R^{4}}
$$

to estimate $\mathcal{A}, \mathcal{B}$ (in (16) and (17)), by changing variables: $(\eta, t)=(x, y, \tau, t) \longmapsto(\tilde{\eta}, \tilde{t})=$ $(\tilde{x}, \tilde{y}, \tilde{\tau}, \tilde{t})$ where

$$
\tilde{x}=R^{-\frac{1}{\theta}} x, \quad \tilde{y}=R^{-\frac{1}{\theta}} y, \quad \tilde{\tau}=R^{-\frac{2}{\theta}} \tau, \quad \tilde{t}=R^{-1} t .
$$

We choose

$$
\Omega=\left\{(\tilde{\eta}, \tilde{t})=(\tilde{x}, \tilde{y}, \tilde{\tau}, \tilde{t}) \in \mathbb{H}^{N} \times \mathbb{R}^{+}: \tilde{\tau}^{2}+|\tilde{x}|^{4}+|\tilde{y}|^{4}+\tilde{t}^{\theta}<2\right\}
$$

Therefore,

$$
\left|\Delta_{\mathbb{H}} \varphi(\tilde{\eta}, \tilde{t})\right| \leq \frac{C}{R^{\frac{2}{\theta}}} \quad \forall(\tilde{\eta}, \tilde{t}) \in \Omega .
$$

As $d \eta d t=R^{\frac{2 N+2}{\theta}+1} d \tilde{\eta} d \tilde{t}$ and $|\eta|_{\mathbb{H}}=R^{\frac{1}{\theta}}|\tilde{\eta}|_{\mathbb{H}}$, we establish the following estimates:

$$
\begin{aligned}
& \int_{Q_{T R}}\left|D_{t / T R}^{q} \varphi\right|^{\beta_{2}^{\prime}}\left(|\eta|_{\mathbb{H}}^{\alpha_{2}} \varphi\right)^{-\frac{\beta_{2}^{\prime}}{\beta_{2}}} d \eta d t \\
& \quad=R^{-q \beta_{2}^{\prime}-\frac{\alpha_{2} \beta_{2}^{\prime}}{\theta \beta_{2}}+\frac{2 N+2}{\theta}+1} \int_{\Omega}\left|D_{\tilde{t} / T}^{q} \Phi \circ \tilde{\rho}\right|^{\beta_{2}^{\prime}}\left(|\tilde{\eta}|_{\mathbb{H}}^{\alpha_{2}} \Phi \circ \tilde{\rho}\right)^{-\frac{\beta_{2}^{\prime}}{\beta_{2}}} d \tilde{\eta} d \tilde{t}
\end{aligned}
$$

and

$$
\begin{aligned}
& \int_{Q_{T R}}\left|\Delta_{\mathbb{H}} \varphi\right|^{\beta_{2}^{\prime}}\left(|\eta|_{\mathbb{H}}^{\alpha_{2}} \varphi\right)^{-\frac{\beta_{2}^{\prime}}{\beta_{2}}} d \eta d t \\
& \leq C R^{-\frac{2}{\theta} \beta_{2}^{\prime}-\frac{\alpha_{2} \beta_{2}^{\prime}}{\theta \beta_{2}}+\frac{2 N+2}{\theta}+1} \int_{\Omega}\left(|\tilde{\eta}|_{\mathbb{H}}^{\alpha_{2}} \Phi \circ \tilde{\rho}\right)^{-\frac{\beta_{2}^{\prime}}{\beta_{2}}} d \tilde{\eta} d \tilde{t} .
\end{aligned}
$$


We choose $\theta$ as the right-hand side of (22) and (23) which are of the same order in $R$. For this purpose, we take $\theta=\frac{2}{q}$, therefore

$$
\mathcal{A} \leq C R^{-q-\frac{q \alpha_{2}}{2 \beta_{2}}+\frac{q}{2} \frac{2 N+2}{\beta_{2}^{\prime}}+\frac{1}{\beta_{2}^{\prime}}}
$$

Similarly, we can get

$$
\mathcal{B} \leq C R^{-q-\frac{q \alpha_{1}}{2 \beta_{1}}+\frac{q}{2} \frac{2 N+2}{\beta_{1}^{\prime}}+\frac{1}{\beta_{1}^{\prime}}}
$$

From (16) and (17), it follows that

$$
\begin{aligned}
& \left(\int_{Q_{T R}}|\eta|_{\mathbb{H}}^{\alpha_{1}}|y|^{\beta_{1}} \varphi d \eta d t\right)^{1-\frac{1}{\beta_{1} \beta_{2}}} \leq C R^{-q-\frac{q \alpha_{2}}{2 \beta_{2}}+\frac{q}{2} \frac{2 N+2}{\beta_{2}^{\prime}}+\frac{1}{\beta_{2}^{\prime}}+\frac{1}{\beta_{2}}\left[-q-\frac{q \alpha_{1}}{2 \beta_{1}}+\frac{q}{2} \frac{2 N+2}{\beta_{1}^{\prime}}+\frac{1}{\beta_{1}^{\prime}}\right]}, \\
& \left(\int_{Q_{T R}}|\eta|_{\mathbb{H}}^{\alpha_{2}}|x|^{\beta_{2}} \varphi d \eta d t\right)^{1-\frac{1}{\beta_{1} \beta_{2}}} \leq C R^{-q-\frac{q \alpha_{1}}{2 \beta_{1}}+\frac{q}{2} \frac{2 N+2}{\beta_{1}^{\prime}}+\frac{1}{\beta_{1}^{\prime}}+\frac{1}{\beta_{1}}\left[-q-\frac{q \alpha_{2}}{2 \beta_{2}}+\frac{q}{2} \frac{2 N+2}{\beta_{2}^{+}}+\frac{1}{\beta_{2}^{\prime}}\right]} .
\end{aligned}
$$

Thus, we have

$$
\left\{\begin{array}{l}
-q-\frac{q \alpha_{2}}{2 \beta_{2}}+\frac{q}{2} \frac{2 N+2}{\beta_{2}^{\prime}}+\frac{1}{\beta_{2}^{\prime}}+\frac{1}{\beta_{2}}\left[-q-\frac{q \alpha_{1}}{2 \beta_{1}}+\frac{q}{2} \frac{2 N+2}{\beta_{1}^{\prime}}+\frac{1}{\beta_{1}^{\prime}}\right]<0, \quad \text { or } \\
-q-\frac{q \alpha_{1}}{2 \beta_{1}}+\frac{q}{2} \frac{2 N+2}{\beta_{1}^{\prime}}+\frac{1}{\beta_{1}^{\prime}}+\frac{1}{\beta_{1}}\left[-q-\frac{q \alpha_{2}}{2 \beta_{2}}+\frac{q}{2} \frac{2 N+2}{\beta_{2}^{\prime}}+\frac{1}{\beta_{2}^{\prime}}\right]<0 .
\end{array}\right.
$$

This condition is equivalent to

$$
Q<Q_{q}^{\bullet}=2\left(1-\frac{1}{q}\right)+\frac{1}{\beta_{1} \beta_{2}-1} \max \left(\left(\alpha_{1}+2\right)+\beta_{1}\left(\alpha_{2}+2\right), \beta_{2}\left(\alpha_{1}+2\right)+\left(\alpha_{2}+2\right)\right) .
$$

Finally, let $R \rightarrow \infty$, taking into account the estimations (14), (17) or (15), (16) and using the Fatou lemma, we get

$$
\begin{aligned}
& \int_{\mathbb{R}^{2 N+1}} \int_{\mathbb{R}^{+}}|\eta|_{\mathbb{H}}^{\beta}|x|^{\beta} d \eta d t \leq 0, \\
& \int_{\mathbb{R}^{2 N+1}} \int_{\mathbb{R}^{+}}|\eta|_{\mathbb{H}}^{\beta}|y|^{\beta} d \eta d t \leq 0 .
\end{aligned}
$$

Therefore, $x \equiv 0$ and $y \equiv 0$, which is a contradiction.

\section{Corollary 3.4 Assume that}

$$
Q<Q_{q}^{\bullet}=2\left(1-\frac{1}{q}\right)+\max \left(X_{1}, X_{2}\right)
$$

where the vector $\left(X_{1}, X_{2}\right)^{T}$ is the solution of the linear system

$$
\left(\begin{array}{cc}
-1 & \beta_{1} \\
\beta_{2} & -1
\end{array}\right)\left(\begin{array}{l}
X_{1} \\
X_{2}
\end{array}\right)=\left(\begin{array}{l}
\alpha_{1}+2 \\
\alpha_{2}+2
\end{array}\right) .
$$

Then there is no weak nontrivial solution $(x, y)$ of the system $\left(\mathrm{FS}_{q}^{2}\right)$. 
Proof To get our result, we use the fact that the vector $\left(X_{1}, X_{2}\right)^{T}$ is given by

$$
\left(\begin{array}{l}
X_{1} \\
X_{2}
\end{array}\right)=\left(\begin{array}{cc}
-1 & \beta_{1} \\
\beta_{2} & -1
\end{array}\right)^{-1}\left(\begin{array}{l}
\alpha_{1}+2 \\
\alpha_{2}+2
\end{array}\right)=\frac{1}{\beta_{1} \beta_{2}-1}\left(\begin{array}{l}
\left(\alpha_{1}+2\right)+\beta_{1}\left(\alpha_{2}+2\right) \\
\beta_{2}\left(\alpha_{1}+2\right)+\left(\alpha_{2}+2\right)
\end{array}\right) .
$$

\section{Systems of $m$ inequalities}

Let $\left(X_{1}, X_{2}, \ldots, X_{m}\right)^{T}$ be the solution of the linear system

$$
\left(\begin{array}{ccccc}
-1 & \beta_{1} & 0 & \ldots & 0 \\
0 & -1 & \beta_{2} & \ddots & \vdots \\
\vdots & \ddots & \ddots & \ddots & 0 \\
0 & 0 & \ddots & \ddots & \beta_{m-1} \\
\beta_{m} & 0 & \ldots & 0 & -1
\end{array}\right)\left(\begin{array}{c}
X_{1} \\
X_{2} \\
\vdots \\
X_{m-1} \\
X_{m}
\end{array}\right)=\left(\begin{array}{c}
\alpha_{1}+2 \\
\alpha_{2}+2 \\
\vdots \\
\alpha_{m-1}+2 \\
\alpha_{m}+2
\end{array}\right),
$$

where $\alpha_{i}$ and $\beta_{i}>1$ are given real numbers, $i \in\{1,2, \ldots, m\}$.

Consider the system

$$
\left(\mathrm{FS}_{q}^{m}\right):\left\{\begin{array}{l}
\mathbf{D}_{0 / t}^{q} x_{i}-\Delta_{\mathbb{H}}\left(\lambda_{i} x_{i}\right) \geq|\eta|^{\alpha_{i+1}}\left|x_{i+1}\right|^{\beta_{i+1}} \\
\left.(\eta, t) \in \mathbb{H}^{N} \times\right] 0,+\infty[, \quad 1 \leq i \leq m \\
x_{m+1}=x_{1}
\end{array}\right.
$$

where $\beta_{m+1}=\beta_{1}, \alpha_{m+1}=\alpha_{1}$, and the initial data are

$$
\left\{\begin{array}{l}
x_{i}(\eta, 0)=x_{i}^{(0)}, \quad 1 \leq i \leq m \\
\frac{\partial x_{i}}{\partial t}(\eta, 0)=x_{i}^{(1)}, \quad 1 \leq i \leq m .
\end{array}\right.
$$

Definition 4.1 Let $\lambda_{i}, i \in\{1,2, \ldots, m\}$ be $m$ bounded measurable functions in $Q_{T}=$ $\mathbb{R}^{2 N+1} \times(0, T)$. A weak solution $\left(x_{1}, \ldots, x_{m}\right)$ of the system $\left(\mathrm{FS}_{q}^{m}\right)$ with positive initial data $\left(x_{i}^{(0)}, x_{i}^{(1)}\right) \in\left(L_{\text {loc }}^{1}\left(\mathbb{R}^{2 N+1}\right)\right)^{2}, i \in\{1,2, \ldots, m\}$, is a vector of locally integrable functions $\left(x_{1}, \ldots, x_{m}\right)$ such that $x_{i} \in L^{\beta_{i}}\left(Q_{T},|\eta|_{\mathbb{H}}^{\alpha_{i}} d \eta d t\right), i \in\{1,2, \ldots, m\}$, satisfying

$$
\left\{\begin{array}{c}
\int_{Q_{T}}\left(-x_{i} D_{t / T}^{q} \varphi+\lambda_{i} x \Delta_{\mathbb{H}} \varphi+|\eta|_{\mathbb{H}}^{\alpha_{i+1}}\left|x_{i+1}\right|^{\beta_{i+1}} \varphi+x_{i}^{(1)}(\eta) D_{t / T}^{q-1} \varphi\right) d \eta d t \\
+\int_{\mathbb{R}^{2 N+1}} x_{i}^{(0)}(\eta) D_{t / T}^{q-1} \varphi(0) d \eta \leq 0, \quad i \in\{1,2, \ldots, m-1\},
\end{array}\right.
$$

and

$$
\left\{\begin{array}{l}
\int_{Q_{T}}\left(-x_{m} D_{t / T}^{q} \varphi+\lambda_{m} x \Delta_{\mathbb{H}} \varphi+|\eta|_{\mathbb{H}}^{\alpha_{1}}\left|x_{1}\right|^{\beta_{1}} \varphi+x_{m}^{(1)}(\eta) D_{t / T}^{q-1} \varphi\right) d \eta d t \\
\quad+\int_{\mathbb{R}^{2 N+1}} x_{m}^{(0)}(\eta) D_{t / T}^{q-1} \varphi(0) d \eta \leq 0
\end{array}\right.
$$

for any nonnegative test function $\varphi \in C_{c}^{2}\left(Q_{T}\right)$, such that $\varphi(\cdot, T)=D_{t / T}^{q-1} \varphi(\cdot, T)=0$.

Theorem 4.2 If the following hypothesis holds:

$$
Q<Q_{q}^{\bullet}=2\left(1-\frac{1}{q}\right)+\max \left(X_{1}, X_{2}, \ldots, X_{m}\right),
$$

then the system $\left(\mathrm{FS}_{q}^{m}\right)$ does not have any weak nontrivial solution. 
Proof The proof is to be reduced to the case $m=3$, the general case can be extended similarly.

Let $\left(x_{1}, x_{2}, x_{3}\right)$ be a nontrivial weak solution of $\left(\mathrm{FS}_{q}^{3}\right)$, as explained in the proof of Theorem 3.3, from the positivity of initial data and $D_{t / T}^{q-1} \varphi \geq 0$, inequalities (28) and (29) imply that

$$
\left\{\begin{array}{l}
\int_{Q_{T R}}|\eta|_{\mathbb{H}}^{\alpha_{1}}\left|x_{1}\right|^{\beta_{1}} \varphi d \eta d t \leq \int_{Q_{T R}} x_{3} D_{t / T R}^{q} \varphi d \eta d t-\int_{Q_{T R}} \lambda_{3} x_{3} \Delta_{\mathbb{H}} \varphi d \eta d t \\
\int_{Q_{T R}}|\eta|_{\mathbb{H}}^{\alpha_{2}}\left|x_{2}\right|^{\beta_{2}} \varphi d \eta d t \leq \int_{Q_{T R}} x_{1} D_{t / T R}^{q} \varphi d \eta d t-\int_{Q_{T R}} \lambda_{1} x_{1} \Delta_{\mathbb{H}} \varphi d \eta d t \\
\int_{Q_{T R}}|\eta|_{\mathbb{H}}^{\alpha_{3}}\left|x_{3}\right|^{\beta_{3}} \varphi d \eta d t \leq \int_{Q_{T R}} x_{2} D_{t / T R}^{q} \varphi d \eta d t-\int_{Q_{T R}} \lambda_{2} x_{2} \Delta_{\mathbb{H}} \varphi d \eta d t .
\end{array}\right.
$$

According to Hölder's inequality, we obtain

$$
\begin{aligned}
& \int_{Q_{T R}}|\eta|_{\mathbb{H}}^{\alpha_{1}}\left|x_{1}\right|^{\beta_{1}} \varphi d \eta d t \leq C\left(\int_{Q_{T R}}|\eta|_{\mathbb{H}}^{\alpha_{3}}\left|x_{3}\right|^{\beta_{3}} \varphi d \eta d t\right)^{\frac{1}{\beta_{3}}} \mathcal{A}_{3}, \\
& \int_{Q_{T R}}|\eta|_{\mathbb{H}}^{\alpha_{2}}\left|x_{2}\right|^{\beta_{2}} \varphi d \eta d t \leq C\left(\int_{Q_{T R}}|\eta|_{\mathbb{H}}^{\alpha_{1}}\left|x_{1}\right|^{\beta_{1}} \varphi d \eta d t\right)^{\frac{1}{\beta_{1}}} \mathcal{A}_{1},
\end{aligned}
$$

and

$$
\int_{Q_{T R}}|\eta|_{\mathbb{H}}^{\alpha_{3}}\left|x_{3}\right|^{\beta_{3}} \varphi d \eta d t \leq C\left(\int_{Q_{T R}}|\eta|_{\mathbb{H}}^{\alpha_{2}}\left|x_{2}\right|^{\beta_{2}} \varphi d \eta d t\right)^{\frac{1}{\beta_{2}}} \mathcal{A}_{2},
$$

where

$$
\begin{aligned}
\mathcal{A}_{i}= & \left(\int_{Q_{T R}}\left|D_{t / T R}^{q} \varphi\right|^{\beta_{i}^{\prime}}\left(|\eta|_{\mathbb{H}}^{\alpha_{i}} \varphi\right)^{-\frac{\beta_{i}^{\prime}}{\beta_{i}}} d \eta d t\right)^{\frac{1}{\beta_{i}^{\prime}}} \\
& +\left(\int_{Q_{T R}}\left|\Delta_{\mathbb{H}} \varphi\right|^{\beta_{i}^{\prime}}\left(|\eta|_{\mathbb{H}}^{\alpha_{i}} \varphi\right)^{-\frac{\beta_{i}^{\prime}}{\beta_{i}}} d \eta d t\right)^{\frac{1}{\beta_{i}^{\prime}}}, \quad i=1,2,3 .
\end{aligned}
$$

From (30), (31), and (32), we get

$$
\begin{aligned}
& \left(\int_{Q_{T R}}|\eta|_{\mathbb{H}}^{\alpha_{1}}\left|x_{1}\right|^{\beta_{1}} \varphi d \eta d t\right)^{1-\frac{1}{\beta_{1} \beta_{2} \beta_{3}}} \leq C \mathcal{A}_{1}^{\frac{1}{\beta_{2} \beta_{3}}} \mathcal{A}_{2}^{\frac{1}{\beta_{3}}} \mathcal{A}_{3}, \\
& \left(\int_{Q_{T R}}|\eta|_{\mathbb{H}}^{\alpha_{2}}\left|x_{2}\right|^{\beta_{2}} \varphi d \eta d t\right)^{1-\frac{1}{\beta_{1} \beta_{2} \beta_{3}}} \leq C \mathcal{A}_{2}^{\frac{1}{\beta_{1} \beta_{3}}} \mathcal{A}_{3}^{\frac{1}{\beta_{1}}} \mathcal{A}_{1}, \\
& \left(\int_{Q_{T R}}|\eta|_{\mathbb{H}}^{\alpha_{3}}\left|x_{3}\right|^{\beta_{3}} \varphi d \eta d t\right)^{1-\frac{1}{\beta_{1} \beta_{2} \beta_{3}}} \leq C \mathcal{A}_{3}^{\frac{1}{\beta_{1} \beta_{2}}} \mathcal{A}_{1}^{\frac{1}{\beta_{2}}} \mathcal{A}_{2} .
\end{aligned}
$$

Applying the test function $\varphi$ (18), and changing of variables (20), given in the proof of Theorem 3.3, we obtain

$$
\mathcal{A}_{i} \leq C R^{\sigma_{i}}, \quad i=1,2,3
$$


such that

$$
\sigma_{i}=-q-\frac{q \alpha_{i}}{2 \beta_{i}}+\frac{q}{2 \beta_{i}^{\prime}} Q+\frac{1}{\beta_{i}^{\prime}}, \quad i=1,2,3
$$

Therefore, from (33), (34), and (35), we get

$$
\begin{aligned}
& \left(\int_{Q_{T R}}|\eta|_{\mathbb{H}}^{\alpha_{1}}\left|x_{1}\right|^{\beta_{1}} \varphi d \eta d t\right)^{1-\frac{1}{\beta_{1} \beta_{2} \beta_{3}}} \leq C R^{\sigma_{3}+\frac{\sigma_{2}}{\beta_{3}}+\frac{\sigma_{1}}{\beta_{2} \beta_{3}}}, \\
& \left(\int_{Q_{T R}}|\eta|_{\mathbb{H}}^{\alpha_{2}}\left|x_{2}\right|^{\beta_{2}} \varphi d \eta d t\right)^{1-\frac{1}{\beta_{1} \beta_{2} \beta_{3}}} \leq C R^{\sigma_{1}+\frac{\sigma_{3}}{\beta_{1}}+\frac{\sigma_{2}}{\beta_{1} \beta_{3}}}, \\
& \left(\int_{Q_{T R}}|\eta|_{\mathbb{H}}^{\alpha_{3}}\left|x_{3}\right|^{\beta_{3}} \varphi d \eta d t\right)^{1-\frac{1}{\beta_{1} \beta_{2} \beta_{3}}} \leq C R^{\sigma_{2}+\frac{\sigma_{1}}{\beta_{2}}+\frac{\sigma_{3}}{\beta_{1} \beta_{2}}} .
\end{aligned}
$$

To end, the exponents of $R$ in (36), (37), and (38) are strictly less than zero if and only if $Q<2(1-1 / q)+\max \left(X_{1}, X_{2}, X_{3}\right)$, where the vector $\left(X_{1}, X_{2}, X_{3}\right)^{T}$ is the solution of

$$
\left(\begin{array}{ccc}
-1 & \beta_{1} & 0 \\
0 & -1 & \beta_{2} \\
\beta_{3} & 0 & -1
\end{array}\right)\left(\begin{array}{l}
X_{1} \\
X_{2} \\
X_{3}
\end{array}\right)=\left(\begin{array}{l}
\alpha_{1}+2 \\
\alpha_{2}+2 \\
\alpha_{3}+2
\end{array}\right)
$$

We conclude that $\left(x_{1}, x_{2}, x_{3}\right) \equiv(0,0,0)$. This contradicts the assertion.

\section{The scalar case}

Let us consider the inequality of the form

$$
\left(\mathrm{FI}_{q}\right):\left\{\begin{array}{l}
\mathbf{D}_{0 / t}^{q}(x)-\Delta_{\mathbb{H}}(\lambda x) \geq|\eta|_{\mathbb{H}}^{\alpha}|x|^{\beta} \quad \text { for }(\eta, t) \in \mathbb{H}^{N} \times \mathbb{R} \\
x(\eta, 0)=x_{0}(\eta) \geq 0, \quad \frac{\partial x}{\partial t}(\eta, 0)=x_{1}(\eta) \geq 0 \quad \text { for } \eta \in \mathbb{H}^{N}
\end{array}\right.
$$

where $\lambda=\lambda(\eta, t)$ is a function defined and measurable in $\mathbb{R}^{2 N+1} \times \mathbb{R}^{+}$and $\alpha, \beta>1, q \in(1,2)$, are real parameters.

Definition 5.1 A local weak solution $x$ of the differential inequality (40) in $Q_{T}=\mathbb{R}^{2 N+1} \times$ $(0, T)$, with positive initial data $x_{0}, x_{1} \in L_{\text {loc }}^{1}\left(\mathbb{R}^{2 N+1}\right)$, is a locally integrable function such that $x \in L^{\beta}\left(Q_{T},|\eta|_{\mathbb{H}}^{\alpha} d \eta d t\right)$ satisfying

$$
\begin{aligned}
& \int_{Q_{T}}\left(-x D_{t / T}^{q} \varphi+\lambda x \Delta_{\mathbb{H}} \varphi+|\eta|_{\mathbb{H}}^{\alpha}|x|^{\beta} \varphi+x_{1}(\eta) D_{t / T}^{q-1} \varphi\right) d \eta d t \\
& \quad+\int_{\mathbb{R}^{2 N+1}} x_{0}(\eta) D_{t / T}^{q-1} \varphi(0) d \eta \leq 0
\end{aligned}
$$

for any nonnegative test function $\varphi \in C_{c}^{2}\left(Q_{T}\right)$ such that $\varphi(\cdot, T)=D_{t / T}^{q-1} \varphi(\cdot, T)=0$.

Remark 5.2 As in Definition 3.1, it is assumed that the integrals in (41) are convergent. In Definition 5.1, if $T=+\infty$, the solution is called global. 
Theorem 5.3 Let $N \geq 1$ and $\beta>1$. Assume that

$$
\alpha>-2 \quad \text { and } \quad 1<\beta<\frac{q(Q+\alpha)+2}{q(Q-2)+2},
$$

then there is no weak nontrivial solution $x$ of the system $\left(\mathrm{FI}_{q}\right)$.

Proof The proof is based on an appropriate choice of the test function. Suppose the problem (40) has a nontrivial global weak solution $x$, let $T, R$, and $\theta>1$ (which will be given later) be three positive reals, let $\varphi$ be a smooth nonnegative test function, since the initial data $x_{0}, x_{1}$ are nonnegative and $D_{t / T}^{q-1} \varphi \geq 0$ (from (8)), then the variational formulation (41) implies

$$
\int_{Q_{T R^{4} / \theta}}|\eta|_{\mathbb{H}}^{\alpha}|x|^{\beta} \varphi d \eta d t \leq \int_{Q_{T R^{4 / \theta}}} x D_{t / T R^{4 / \theta}}^{q} \varphi d \eta d t-\int_{Q_{T R^{4 / \theta}}} \lambda x \Delta_{\mathbb{H}} \varphi d \eta d t .
$$

The test function $\varphi$ should be given to ensure that

$$
\int_{Q_{T R} 4 / \theta}\left(\left|D_{t / T}^{q} \varphi\right|^{\beta^{\prime}}+\left|\Delta_{\mathbb{H}} \varphi\right|^{\beta^{\prime}}\right)\left(|\eta|_{\mathbb{H}}^{\alpha} \varphi\right)^{-\beta^{\prime} / \beta} d \eta d t<\infty
$$

To estimate the right side of (43), we apply Young's inequality for an arbitrary $\varepsilon>0$, we have

$$
\begin{aligned}
\int_{Q_{T R^{4 / \theta}}} x D_{t / T R^{4 / \theta}}^{q} \varphi d \eta d t= & \int_{Q_{T R^{4 / \theta}}} x\left(|\eta|_{\mathbb{H}}^{\alpha} \varphi\right)^{\frac{1}{\beta}}\left(|\eta|_{\mathbb{H}}^{\alpha} \varphi\right)^{-\frac{1}{\beta}} D_{t / T R^{4 / \theta}}^{q} \varphi d \eta d t \\
\leq & \varepsilon \int_{Q_{T R^{4} / \theta}}|\eta|_{\mathbb{H}}^{\alpha}|x|^{\beta} \varphi d \eta d t \\
& +C_{\varepsilon} \int_{Q_{T R^{4 / \theta}}}\left|D_{t / T R^{4 / \theta}}^{q} \varphi\right|^{\beta^{\prime}}\left(|\eta|_{\mathbb{H}}^{\alpha} \varphi\right)^{-\frac{\beta^{\prime}}{\beta}} d \eta d t
\end{aligned}
$$

and

$$
\begin{aligned}
\int_{Q_{T R^{4} / \theta}} \lambda x \Delta_{\mathbb{H}} \varphi d \eta d t= & \int_{Q_{T R^{4 / \theta}}} \lambda x\left(|\eta|_{\mathbb{H}}^{\alpha} \varphi\right)^{\frac{1}{\beta}}\left(|\eta|_{\mathbb{H}}^{\alpha} \varphi\right)^{-\frac{1}{\beta}} \Delta_{\mathbb{H}} \varphi d \eta d t \\
\leq & \varepsilon \int_{Q_{T R^{4} / \theta}}|\eta|_{\mathbb{H}}^{\alpha}|x|^{\beta} \varphi d \eta d t \\
& +C_{\varepsilon}\|\lambda\|_{\infty}^{\beta^{\prime}} \int_{Q_{T R^{4} / \theta}}\left|\Delta_{\mathbb{H}} \varphi\right|^{\beta^{\prime}}\left(|\eta|_{\mathbb{H}}^{\alpha} \varphi\right)^{-\frac{\beta^{\prime}}{\beta}} d \eta d t .
\end{aligned}
$$

By considering $\varepsilon$ small enough, we have

$$
\int_{Q_{T R^{4} / \theta}}|\eta|_{\mathbb{H}}^{\alpha}|x|^{\beta} \varphi d \eta d t \leq C_{\varepsilon} \int_{Q_{T R^{4} / \theta}}\left(\left|D_{t / T R^{4 / \theta}}^{q} \varphi\right|^{\beta^{\prime}}+\left|\Delta_{\mathbb{H}} \varphi\right|^{\beta^{\prime}}\right)\left(|\eta|_{\mathbb{H}}^{\alpha} \varphi\right)^{-\frac{\beta^{\prime}}{\beta}} d \eta d t
$$

Take

$$
\varphi(\eta, t)=\varphi(x, y, \tau, t)=\Phi\left(\frac{\tau+|x|^{2}+|y|^{2}+t^{\theta}}{R^{4}}\right),
$$


where $\Phi \in \mathcal{D}\left(\mathbb{R}^{+}\right)$, which satisfies $0 \leq \Phi \leq 1$ and (19), therefore

$$
\Delta_{\mathbb{H}} \varphi(\eta, t)=\frac{4 N \Phi^{\prime}(\rho)}{R^{4}}+\frac{8 \Phi^{\prime \prime}(\rho)}{R^{8}}\left[|x|^{2}+|y|^{2}\right]
$$

where

$$
\rho=\frac{\tau+|x|^{2}+|y|^{2}+|t|^{\theta}}{R^{4}} .
$$

To estimate the right-hand side in (44), we again change the variables,

$$
\tilde{t}=R^{-4 / \theta} t, \quad \tilde{\tau}=R^{-4} \tau, \quad \tilde{x}=R^{-2} x, \quad \tilde{y}=R^{-2} y,
$$

we put

$$
\tilde{\rho}=\tilde{\tau}+|\tilde{x}|^{2}+|\tilde{y}|^{2}+\tilde{t}^{\theta}
$$

To guarantee that $\operatorname{supp} \Phi \subseteq \Omega$, we assume that

$$
\Omega=\left\{(\tilde{\eta}, \tilde{t})=(\tilde{x}, \tilde{y}, \tilde{\tau}, \tilde{t}) \in \mathbb{R}^{2 N+1} \times \mathbb{R}, \tilde{\rho} \leq 2\right\} .
$$

Therefore,

$$
\left|\Delta_{\mathbb{H}} \varphi(\tilde{\eta}, \tilde{t})\right| \leq \frac{C}{R^{4}} \quad \forall(\tilde{\eta}, \tilde{t}) \in \Omega
$$

from $d \eta d t=R^{4 N+4+4 / \theta} d \tilde{\eta} d \tilde{t},|\eta|_{\mathbb{H}}=R^{2}|\tilde{\eta}|_{\mathbb{H}}$, and $\left|D_{t / T R^{4 / \theta}}^{q} \varphi\right|=R^{\frac{-4 q}{\theta}}\left|D_{t / T}^{q} \varphi\right|$, we have (44) so that

$$
\begin{aligned}
& \int_{Q_{T R^{4 / \theta}}}\left|\Delta_{\mathbb{H}} \varphi\right|^{\beta^{\prime}}\left(|\eta|_{\mathbb{H}}^{\alpha}|x|^{\beta}\right)^{-\frac{\beta^{\prime}}{\beta}} d \eta d t \\
& \quad \leq R^{-4 \beta^{\prime}+4 N+4+\frac{4}{\theta}-2 \alpha \frac{\beta^{\prime}}{\beta}} \int_{\Omega}\left|\Delta_{\mathbb{H}} \Phi \circ \tilde{\rho}\right|^{\beta^{\prime}}\left(|\tilde{\eta}|_{\mathbb{H}}^{\alpha} \Phi \circ \tilde{\rho}\right)^{-\frac{\beta^{\prime}}{\beta}} d \tilde{\eta} d \tilde{t}
\end{aligned}
$$

and

$$
\begin{aligned}
& \int_{Q_{T R^{4} / \theta}}\left|D_{t / T R^{4 / \theta}}^{q} \varphi\right|^{\beta^{\prime}}\left(|\eta|_{\mathbb{H}}^{\alpha}|x|^{\beta}\right)^{-\frac{\beta^{\prime}}{\beta}} d \eta d t \\
& \quad \leq R^{-\frac{4 q}{\theta} \beta^{\prime}+4 N+4+\frac{4}{\theta}-2 \alpha \frac{\beta^{\prime}}{\beta}} \int_{\Omega}\left|D_{t / T}^{q} \Phi \circ \tilde{\rho}\right|^{\beta^{\prime}}\left(|\tilde{\eta}|_{\mathbb{H}}^{\alpha} \Phi \circ \tilde{\rho}\right)^{-\frac{\beta^{\prime}}{\beta}} d \tilde{\eta} d \tilde{t} .
\end{aligned}
$$

For the same exponent of $R$ in (47) and (48), it is convenient to write $\theta=q$, then

$$
\int_{Q_{T R} 4 / q}|\eta|_{\mathbb{H}}^{\alpha}|x|^{\beta} \varphi d \eta d t \leq C R^{-4 \beta^{\prime}+4 N+4+\frac{4}{q}-2 \alpha \frac{\beta^{\prime}}{\beta}}
$$

where

$$
C=C_{\varepsilon} \int_{\Omega}\left(\left|D_{t / T}^{q} \Phi \circ \tilde{\rho}\right|^{\beta^{\prime}}+\left|\Delta_{\mathbb{H}} \Phi \circ \tilde{\rho}\right|^{\beta^{\prime}}\right)\left(|\tilde{\eta}|_{\mathbb{H}}^{\alpha} \Phi \circ \tilde{\rho}\right)^{-\frac{\beta^{\prime}}{\beta}} d \tilde{\eta} d \tilde{t}
$$


In the case that

$$
1<\beta<\frac{q(Q+\alpha)+2}{q(Q-2)+2},
$$

the exponent of $R$ in (49) is negative, it means that $R \longrightarrow+\infty$ is qualified to apply Fatou's lemma to get

$$
\int_{0}^{\infty} \int_{\mathbb{R}^{2 N+1}}|\eta|_{\mathbb{H}}^{\alpha}|x|^{\beta} d \eta d t=0
$$

Thus, $x \equiv 0$, and this contradicts the fact that $x$ is a nontrivial solution of (40).

Remark 5.4 The positivity condition on the initial data can be weakened and replaced by

$$
\int_{Q_{T}} x_{1}(\eta) D_{t / T}^{q-1} \varphi d \eta d t+\int_{\mathbb{R}^{2 N+1}} x_{0}(\eta) D_{t / T}^{q-1} \varphi(0) d \eta \geq 0
$$

Remark 5.5 The assertion $\alpha>-2$ and $1<\beta<\frac{q(Q+\alpha)+2}{q(Q-2)+2}$ is equivalent to $Q<2\left(1-\frac{1}{q}\right)+\frac{\alpha+2}{\beta-1}$, which motivates that Theorem 5.3 is a special case of Theorem 4.2 (in other words $\left(\mathrm{FI}_{q}\right) \equiv$ $\left.\left(\mathrm{FS}_{q}^{1}\right)\right)$.

Remark 5.6 $q=2$ covers the case of a hyperbolic inequality of the type

$$
\frac{\partial^{2} x}{\partial t^{2}}-\Delta_{\mathbb{H}}(\lambda x) \geq|\eta|_{\mathbb{H}}^{\alpha}|x|^{\beta}
$$

studied by Pohozaev and Véron [3].

Remark 5.7 By assuming $q \rightarrow \infty$, then it is easy to find the well-known critical exponent $\beta_{\infty}=\frac{Q+\alpha}{Q-2}$ for the elliptic inequalities $[3,23]$.

\section{Competing interests}

The authors declare that they have no competing interests.

Authors' contributions

Each of the authors contributed to each part of this study equally and approved the final version of the manuscript.

\section{Author details}

'Department of Mathematics, Constantine University, Constantine, 25000, Algeria. ${ }^{2}$ Department of Mathematics, University of El-Oued, El-Oued, 39000, Algeria. ${ }^{3}$ LAMIS Laboratory, Department of Mathematics, Tebessa University, Tebessa, 12000, Algeria. ${ }^{4}$ Department of Mathematics, Guelma University, Guelma, 24000, Algeria.

\section{Acknowledgements}

The authors would like to express their deepest gratitude to Prof. Dumitru Baleanu and reviewers for their valuable comments.

Received: 12 October 2016 Accepted: 22 December 2016 Published online: 12 January 2017

\section{References}

1. El Hamidi, A, Kirane, M: Nonexistence results of solutions to systems of semilinear differential inequalities on the Heisenberg group. Abstr. Appl. Anal. 2004(2), 155-164 (2004)

2. El Hamidi, A, Obeid, A: Systems of semilinear higher order evolution inequalities on the Heisenberg group. J. Math. Anal. Appl. 208(1), 77-90 (2003)

3. Pohozaev, S, Véron, L: Nonexistence results of solutions of semilinear differential inequalities on the Heisenberg group. Manuscr. Math. 102, 85-99 (2000)

4. Fujita, $\mathrm{H}$ : On the blowing up of solutions of the Cauchy problem for $u_{t}=\Delta u+u^{1+\alpha}$. J. Fac. Sci., Univ. Tokyo, Sect. I 13, 109-124 (1966) 
5. Kilbas, AA, Srivastava, HM, Trujillo, JJ: Theory and Applications of Fractional Differential Equations. Elsevier, Amsterdam (2006)

6. Kirane, M, Laskri, Y, Tatar, NE: Critical exponents of Fujita type for certain evolution equations and systems with spatio-temporal fractional derivatives. J. Math. Anal. Appl. 312, 488-511 (2005)

7. Bordoni, S, Filippucci, R, Pucci, P: Nonlinear elliptic inequalities with gradient terms on the Heisenberg group. Nonlinear Anal., Theory Methods Appl. 121, 262-279 (2015)

8. Sitho, S, Ntouyas, SK, Yukunthorn, W, Tariboon, J: Lyapunov's type inequalities for hybrid fractional differential equations. J. Inequal. Appl. 2016, 170 (2016)

9. Atangana, A, Baleanu, D: New fractional derivatives with nonlocal and non-singular kernel: theory and application to heat transfer model. Therm. Sci. 20(2), 763-769 (2016)

10. Atangana, A, Koca, I: Chaos in a simple nonlinear system with Atangana-Baleanu derivatives with fractional order. Chaos Solitons Fractals 89, 447-454 (2016)

11. Atangana, A: Derivative with two fractional orders: a new avenue of investigation toward revolution in fractional calculus. Eur. Phys. J. Plus 131(10), 373 (2016)

12. Samko, SG, Kilbas, AA, Marichev, OL: Fractional Integrals and Derivatives Theory and Applications. Gordon \& Breach, Yverdon (1993)

13. Podlubny, I: Fractional Differential Equations. Mathematics in Science and Engineering. Academic Press, New York (1999)

14. Abdeljawad, T, Baleanu, D: Discrete fractional differences with nonsingular discrete Mittag-Leffler kernels. Adv. Differ. Equ. 2016, 232 (2016)

15. Debbouche, A, Baleanu, D, Agarwal, RP: Nonlocal nonlinear integrodifferential equations of fractional orders. Bound. Value Probl. 2012, 78 (2012)

16. Suganya, S, Baleanu, D, Kalamani, P, Arjunan, MM: On fractional neutral integro-differential systems with state-dependent delay and non-instantaneous impulses. Adv. Differ. Equ. 2015, 372 (2015)

17. Heinonen, J: Calculus on Carnot groups. Report, vol. 68, 1-31, Fall School in Analysis (1995)

18. Capogna, L, Danielli, D, Pauls, SD, Tyson, JT: An Introduction to the Heisenberg Group and the Sub-Riemannian Isoperimetric Problem. Birkhäuser, Berlin (2007)

19. Folland, G: Harmonic Analysis in Phase Space, vol. 122. Princeton University Press, New York (1989)

20. Heinonen, J: Lectures on Analysis on Metric Spaces. Springer, New York (2001)

21. Semmes, S: An introduction to Heisenberg groups in analysis and geometry. Not. Am. Math. Soc. 50(6), 640-646 (2003)

22. Stein, EM: Harmonic Analysis: Real Variable Methods, Orthogonality and Oscillatory Integrals. Princeton University Press, Princeton (1993)

23. Brindelli, I, Capuzzo Dolcetta, I, Cutri, A: Liouville theorem for semilinear equation on the Heisenberg group. Ann. Inst. Henri Poincaré, Anal. Non Linéaire 14(3), 295-308 (1997)

\section{Submit your manuscript to a SpringerOpen ${ }^{\circ}$ journal and benefit from:}

$\checkmark$ Convenient online submission

Rigorous peer review

- Immediate publication on acceptance

Open access: articles freely available online

- High visibility within the field

- Retaining the copyright to your article 